\author{
Military Technical College \\ Kobry El-Kobbah, \\ Cairo, Egypt
}

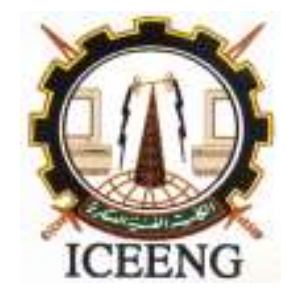

\section{$11^{\text {th }}$ International Conference on Electrical Engineering ICEENG 2018}

\title{
Application of Adaptive Recovery CAMP Reconstruction Algorithm in Compressing Sensing Radar Signal Processing
}

\author{
M. H. Hossiny", Sameh G. Salem*, Fathy M. Ahmed", and K. H. Moustafa*
}

\begin{abstract}
Compressive Sensing (CS) theory uses the sparsity property in pulsed radar signal to reduce the number of measurements needed for digital acquisition, which causes reduction in the size, weight, power consumption, and cost [1-3]. A proposed adaptive recovery Complex Approximate Massage Passing (CAMP) reconstruction algorithm was introduced by the authors in [4] to enhance the detection performance of pulsed radar receiver compared to the traditional CAMP algorithm. In the present work, this proposed algorithm is used in pulsed radar receiver to resolve the limitation of the number of detected targets in the traditional CAMP algorithm with a remarkable reduction in time complexity.
\end{abstract}

\section{Keywords}

Compressive Sensing,CAMP algorithm, Adaptive threshold.

* Egyptian Armed Forces. 


\section{Introduction}

In recent years, because of people's growing demand for information, the bandwidth of the signal carrying the information becomes wider. Digital signal processing (DSP) plays an important role in signal enhancement due to the technology and the rapid development of DSP. Sampling is the only way to convert the analog signal into digital signal, and sampling theorem is a bridge which links between them. Sampling theorem (Shannon theorem) demands that the sampling rate should equal at least twice the bandwidth of the signal, in order to reconstruct the original analog signal without distortion or aliasing.

In order to be able to accurately probe targets, have a good radar resolution, and to reduce effect of jamming, large-bandwidth and high dynamic range signal should be launched, which requires a very high sampling rate and high speed Analog-to-Digital converter(ADC).

Currently available ADC and signal processors technology is a limiting factor in designing of wideband (high-resolution) radar systems, because in many cases the required performance is beyond what is either technologically possible or too expensive. In [4], Donohue and Candes proposed Compressive Sensing (CS) theory, which showed that a signal having a sparse representation can be recovered exactly from a small number or random sets [5]. Applying CS in radar receivers has many advantages such as reduction in sampling rate, low cost and less processing time compared with uncompressed radar receivers.

CS theory combines the sampling and compression to reduce the signal sampling rate, the cost of the transmission, and the processing time. When the signal has the characteristic of sparsity, the original radar signal can be exactly reconstructed from under-sampled measurements [6].

Considering the problem of recovering a sparse signal, $x$, with a number of samples (measurements), $M$, the simplest way to reduce the number of sparse samples, $N$, is the linear operator where the measurement vector, $y$, is given by.

$$
\begin{aligned}
& y=A x+n \quad \text { and, } \\
& \delta=M / N \quad, \quad \rho=k / M
\end{aligned}
$$

Where, $A$, is an $(M \times N)$ sensing matrix, $x$, is a $(N \times 1)$ sparse radar signal, $n$, is Gaussian random noise with zero mean and unity variance, $\rho$, is the radar signal sparsity with non-zero elements,$k$, and with under-sampling factor is $\delta$. Fig. (1), shows the general CS diagram, which explain the process of compression and reconstruction of radar signal.

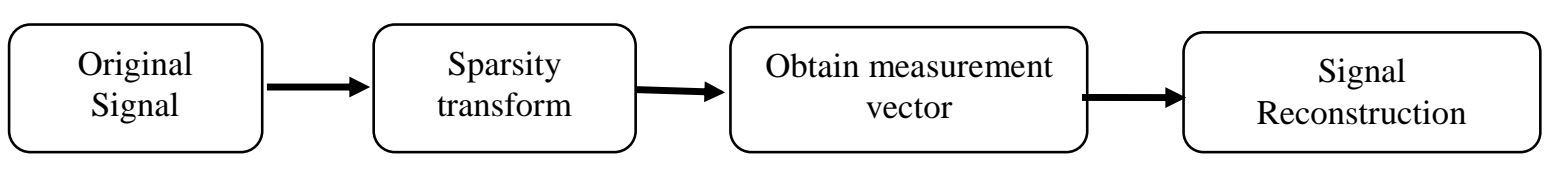

Fig. 1. General block diagram of compressive sensing [7]. 
As shown in Fig. (1), application of CS in radar signal processing may be organized separately in three aspects: sparse representation of radar signal, designing of sensing matrix, and reconstruction algorithms of the radar signal [8]. Two properties of matrix $A$ should be achieved in the previous three aspects to ensure that the reconstruction process will not fail. These properties are; incoherence and the Restricted I sometry Property (RIP) $[9,10]$. The sensing matrix, $A$, is designed to ensure that the sparse signal, $x$, can be reconstructed perfectly. Hence, the structure of the sensing matrix must be incorporated in the recovery algorithm or otherwise the recovery will not be successful. First, (RIP), is used to control the two kinds of errors due to the radar system noise which is induced inside the receiver itself and Mean Square Error (MSE) which is induced due to the reconstruction process. To insure that the recovery process is stable in presence of the noise (uniqueness), and to control the tolerant for both types of errors the RIPis given by [11]:

$$
(1-\gamma)\|x\|_{2}^{2} \leq\|\mathrm{A} \cdot x\|_{2}^{2} \leq(1+\gamma)\|x\|_{2}^{2}
$$

Where $\gamma$ is a fixed number less than unity.

First problem, is the complexity of the reconstruction algorithms due to RIP, which controls the tolerance of the MSE between the original and the reconstructed signal. This tolerance is obtained during the reconstruction process because of updating the residual. This the iteration during the reconstruction process. Finally, incoherence property of the matrix, $A$, is used to determine the minimum number of measurements, or the reduction factor in the received radar signal. It can be achieved in the radar signal without effecting on the performance of the recovery process, by determining the number of measurements, $M$, according to the following relation [12]:

$$
\mathrm{M} \geq \mathrm{k}^{2} \ln \mathrm{N}
$$

Second problem, is the limitation of the detected targets number in the received radar signal according to the incoherence property. These problems will be resolved in the present work.

This paper is organized as follows; after the introduction, section 2 gives a survey on CAMP algorithm. Section 3 focuses on the proposed Adaptive recovery CAMP algorithm. The simulation results, and the performance evaluation through the ROC curves of the CAMP algorithm compared to the proposed Adaptive recovery CAMP algorithm is presented in section 4. Finally, conclusion comes in section 5 .

\section{Complex Approximate Message Passing (CAMP) Algorithm}

CAMP algorithm is one of the most successful algorithms for the CS reconstruction [13], which provides a simple and fast converging iterative method for solving complex-lasso. CAMP algorithm has been chosen for reconstructing the radar signal, as it is faster and simpler than both $\ell 1$-norm minimization and the Orthogonal Matched Pursuit(OMP) algorithms according to Pseudo code as shown in Fig.(2).The flow chart of the CAMP algorithm is illustrated in Fig. (3) [14]. 


\section{CAMP algorithm}

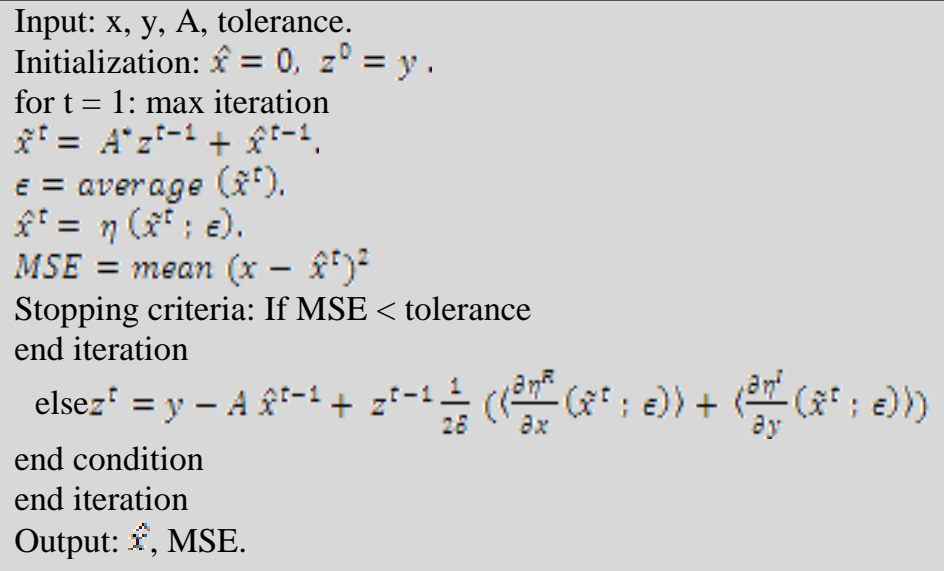

Fig. 2. Pseudo code of the CAMP algorithm.

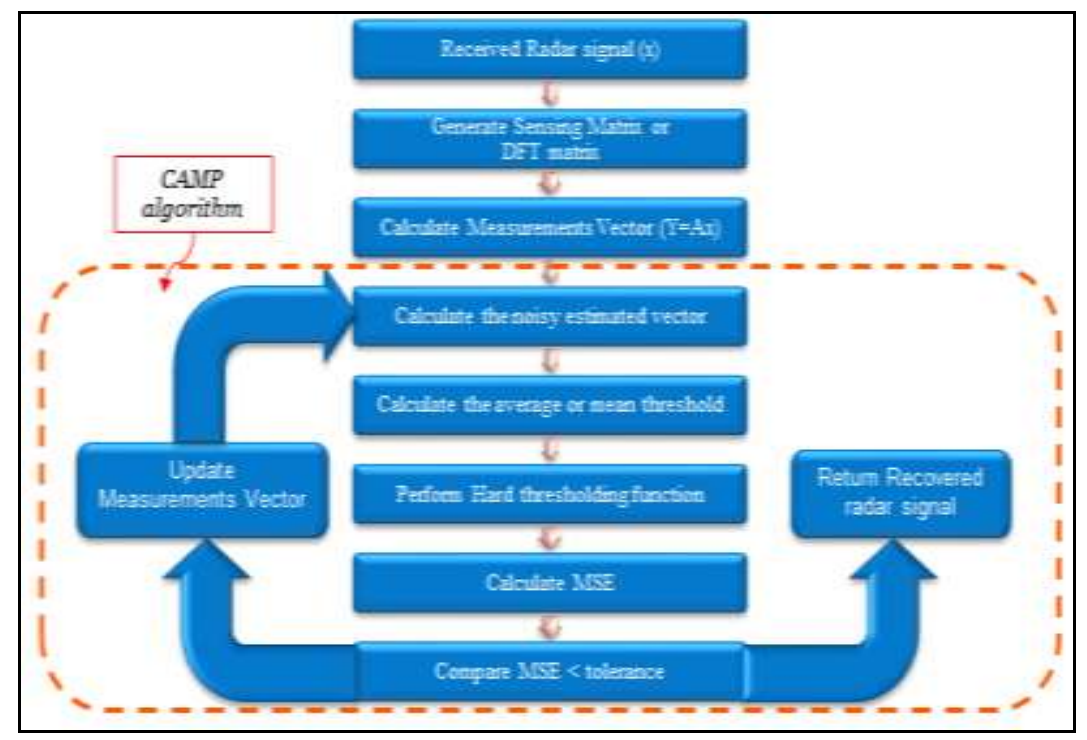

Fig. 3. Flow chart of CAMP algorithm based on the CS theory [13].

In many related works, the threshold value is calculated according to the mean or the average of the noisy estimated radar signal, so it is selected to be a constant value [15-17]. Suppose a pulsed radar signal with length $(N)$ equals 1024 samples, this radar signal can be compressed with under sampling factor $=0.75$, so the number of measurements, $M=256$ samples according to the incoherence property given by Equ (4), so the maximum number of targets (nonzero elements) can be reconstructed are only six targets. Fig. (4),shows the detection of six targetsat $\mathrm{SNR}=10 \mathrm{~dB}$ before and after reconstruction. 

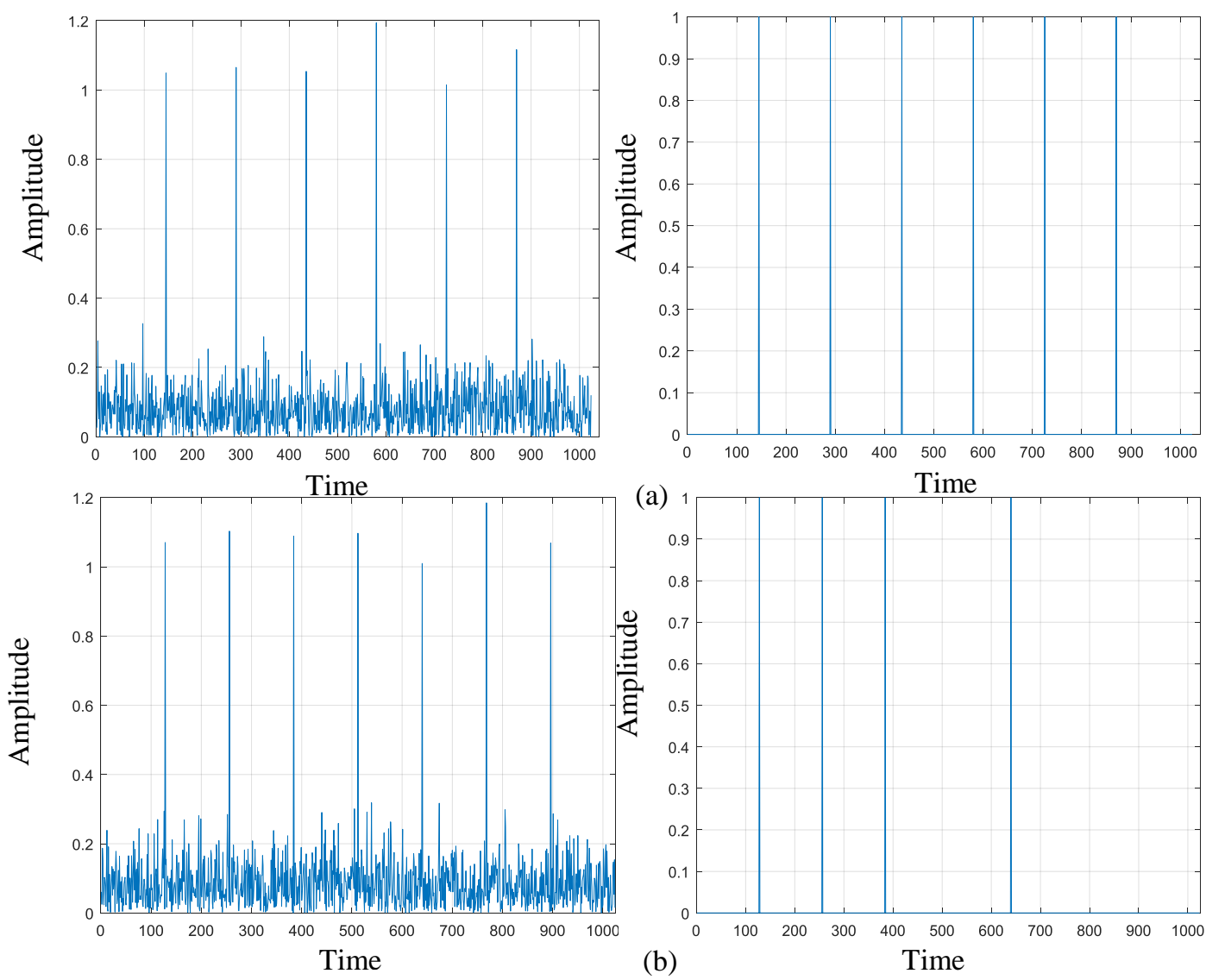

Fig. 4. Simulation Results of CAMP algorithm reconstruction based on CS at $\mathrm{SNR}=10 \mathrm{~dB}$ for (a) six targets, and (b) seven targets.

As shown in Fig. (4.a), the CAMP algorithm can recover the received radar signal, as the number of targets did not excess the number of nonzero elements that had been calculated using the incoherence property. In case of, number of targets excesses the number of nonzero elements the CAMP algorithm cannot recover the radar signal, as shown in Fig. (4.b), the CAMP algorithm failed to reconstruct it. Most of the existing compressive sensing recovery algorithms have a threshold or a regularization parameter, which affects the quality of the reconstruction result. In the present work, a suggested method for calculating the threshold, which is used inside the recovery algorithms of CS theory is used, in order to manage the two problems that have been discussed before. An adaptive method is introduced to calculate the threshold that is used in the recovery algorithm to perform a hard Thresholding function or smoothing the estimated radar signal during reconstruction process. As a result, the CAMP algorithm can be modified in order to give a better performance during recovering the low SNR radar signal by calculating its threshold adaptively. This proposed recovery algorithm is called Adaptive recovery Complex Approximate Message Passing (Adaptive recovery CAMP) algorithm, which shall be discussed in the next section. 


\section{Adaptive Recovery CAMP Algorithm}

The threshold in the proposed algorithm is calculated adaptively by estimating the smallest level in a window of $M$ range cells for the noisy estimation of the radar signal [18]. The adaptive threshold is used to increase the number of detected targets in the received radar signal, than the traditional CAMP algorithm detects in recovery process of radar signal.

The proposed Adaptive recovery CAMP algorithm can reconstruct the radar signal in three steps; firstly, the estimated signal is computed by multiplying the measurements vector by the transpose of the DFT matrix, $A^{T}$. Then, the threshold is calculated by adaptive method according to the value of noisy estimation of radar signal, $\tilde{x}$, based on the smallest window. Finally, the estimated signal is refined and smoothed by using the iterative thresholding function to obtain the estimated signal, $\widehat{x}$. Fig. (5), shows Pseudo code of the proposed Adaptive recovery CAMP algorithm.

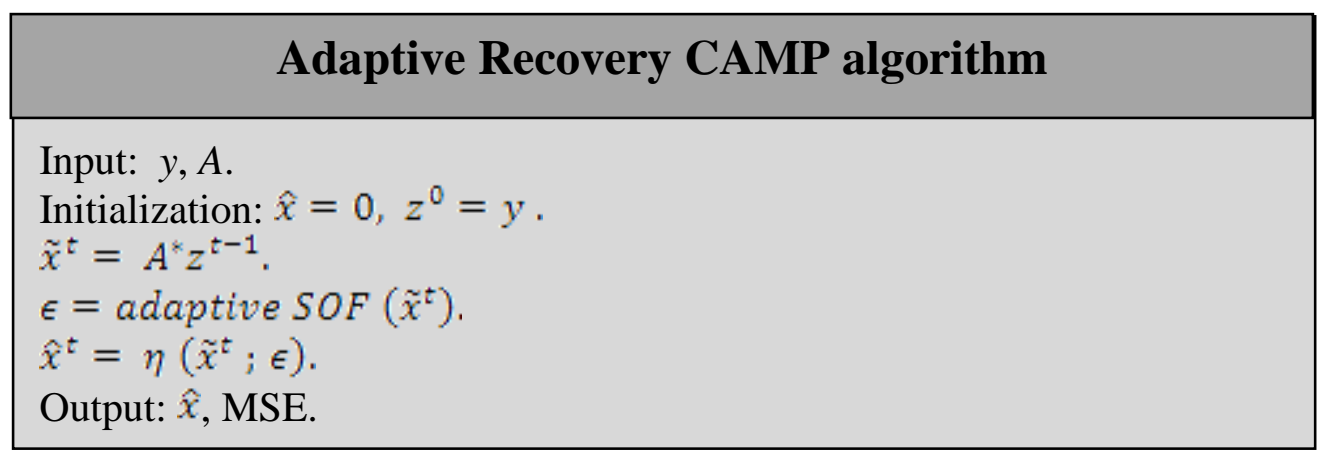

Fig. 5. Pseudo code of the proposed Adaptive recovery CAMP algorithm.

As shown in Fig. (5), the input to the proposed Adaptive recovery CAMP algorithm is the DFT matrix, $\mathrm{A}$, and the measurement vector, $\mathrm{y}$. The vector, $\mathrm{y}$, indicates to the measurement vector or the under-sampled radar signal (received radar signal with lower number of samples) which is used to reconstruct the received radar signal. A, represents the DFT matrix of the CS theory which is used to obtain the measurement vector and to estimate the signal. Fig. (6), shows the flow chart of the Adaptive recovery CAMP algorithm. 


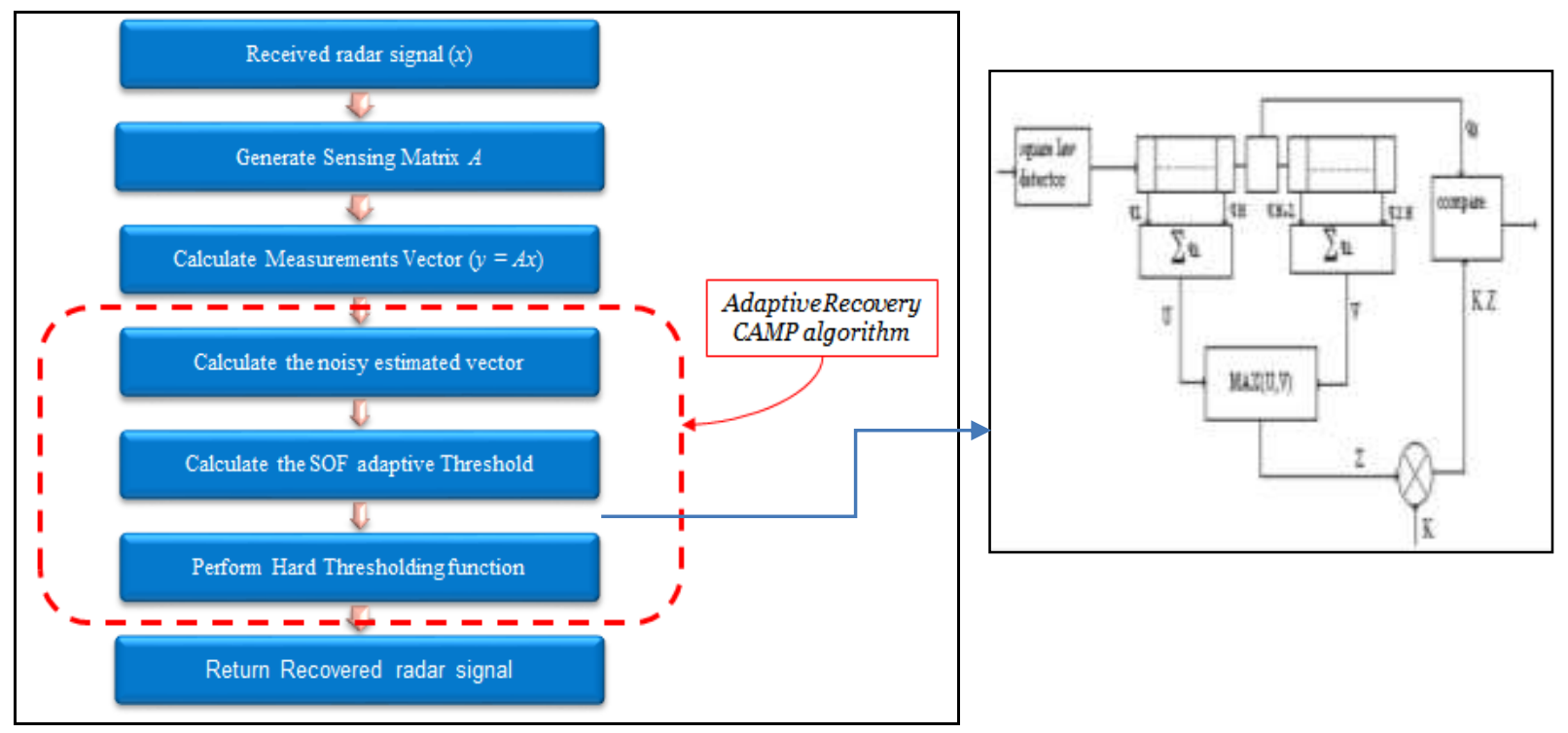

Fig. 6. Flow chart of adaptive recovery CAMP algorithm based on CS.

The adaptive threshold is an optimum method (maximizes detection probability) in a homogeneous background when the reference cells contain Independent and Identically Distributed observations governed by an exponential distribution [18]. As the size of the reference window increases, the detection probability approaches that of the optimum detector, which is based on a fixed threshold. The noisy estimated signal is divided into many range cells and at every test cell a comparison between the summation of lead 5 range cell and the summation of lag 5 range cell is performed, then the threshold at this cell is chosen according to the comparator module. If the comparator module chooses the largest of summation of range cells, so the adaptive threshold is called the Greatest of (GOF), and If the comparator module chooses the least of summation of range cells, so the adaptive threshold is called the Smallest of them (SOF). A very important factor that is called the multiplication factor which is used to tuning the level of the adaptive threshold according to the required value of the probability of false alarms. The proposed adaptive recovery CAMP algorithm can recover the pulsed radar signal in three steps; calculate the noisy estimated signal, $\tilde{x}$, determine the adaptive threshold, and smooth the noisy estimated signal, $\tilde{x}$, to obtain the estimated radar signal, $\widehat{x}$.

\section{Simulation Results}

The simulation results are done for testing the proposed algorithm compared with that of traditional algorithm, from three points of view; the number of detected targets, the resolution performance of the proposed algorithm, and the time complexity evaluation. Suppose a radar signal 1024 samples $(N=1024)$, that can be compressed to 256 samples $(M=256)$, so the number of non-zero coefficients for traditional algorithm can be calculated using equation (4). For the proposed algorithm, Equ (4) can be modified to equation (5) according to the trail of simulation results.

$$
\mathrm{M} \geq \text { const } * \mathrm{k}^{2} \ln \mathrm{N}
$$




\subsection{Number of detected targets}

Fig. (7), shows the reconstructing simulation of the pulsed radar signal with six, seven, and eight targets in the received radar signals according to the incoherence property by using the proposed adaptive recovery CAMP algorithm. It's illustrated that, there is no limitation on the number of detected targets in the proposed adaptive recovery CAMP algorithm as the traditional CAMP algorithm, which is limited to six targets with the same radar parameters and the same compression ratio.
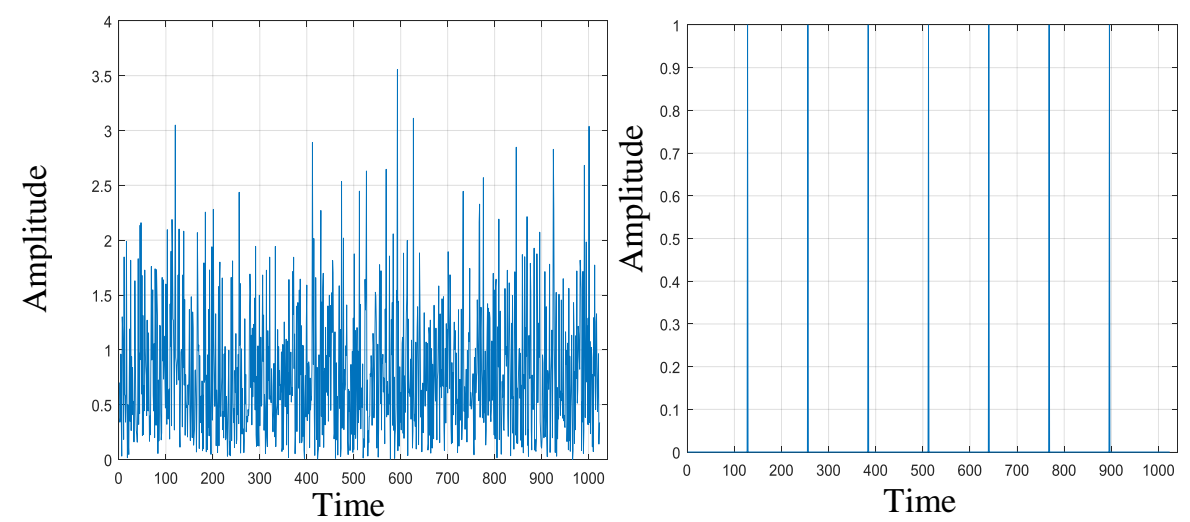

Fig. 7. Simulation results for reconstructing the radar signal with seven targets using the proposed at $\mathrm{SNR}=0 \mathrm{~dB}, \mathrm{Pfa}=10^{-6}$, and $\delta=0.75$ a pulsed radar signal with seven targets.

\subsection{Resolution of the proposed algorithm}

The range resolution of the proposed algorithm is evaluated by simulated two targets at range cells number (512), and (513) respectively.Fig. (8), shows the simulation results for the resolution performance using the proposed Adaptive recovery CAMP algorithmat $\mathrm{SNR}=0 \mathrm{~dB}, \mathrm{Pfa}=10^{-6}$, and reduction ratio $(\delta=0.75)$.

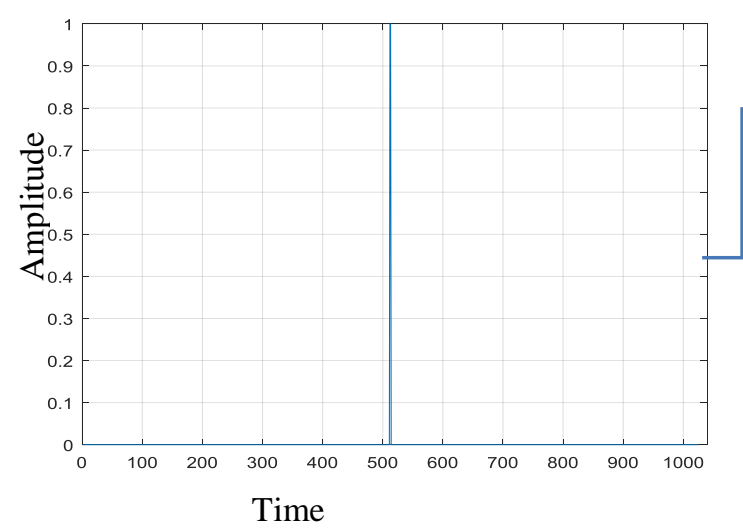

(a)

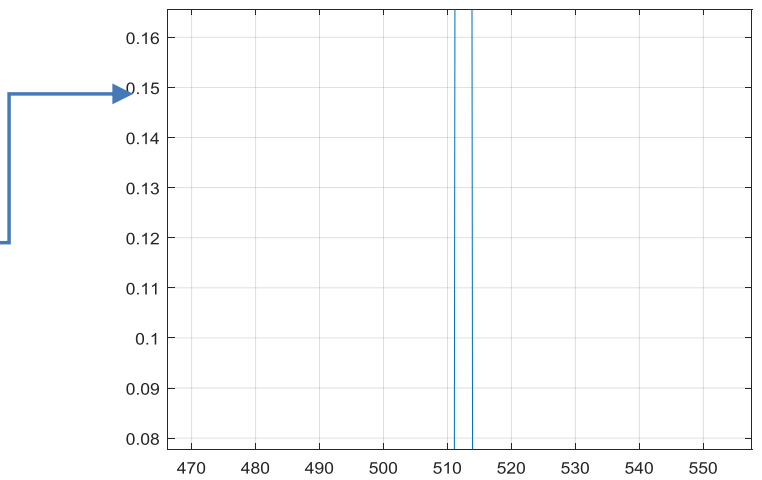

(b)

Fig. 8. Resolution in range for two successive targets using the proposed algorithmat $\mathrm{SNR}=0 \mathrm{~dB}, \mathrm{P}_{\mathrm{fa}}=10^{-6}$, and $\delta=0.75$,

(a) Reconstructed radar signal. (b)Zooming in reconstructed radar signal. 


\subsection{Time complexity performance}

The time complexity of the proposed approach is compared with that of the traditional CAMP taken through the reconstruction process. This comparison is performed at reduction ratio of $75 \%$ in range samples for the both approaches, as shown in table (1):

Table (1) Time complexity comparison between Traditional CAMP algorithm and proposed adaptive recovery CAMP algorithm at $75 \%$ reduction ratio in range sweeps.

\begin{tabular}{|l|c|}
\hline \multicolumn{1}{|c|}{ Comparison aspects } & Time of Processing (sec) \\
\hline Traditional CAMP algorithm & Around 2.354 \\
\hline Proposed adaptive recovery CAMP algorithm & 0.450 \\
\hline
\end{tabular}

From this table, it is found that, the processing time as well as the complexity of the proposed algorithm is smaller than that of the traditional algorithm because of the traditional CAMP algorithm can recover the radar signal in five steps or iterations until the MSE become lower than the tolerance that specified by the Restricted Isometry Property (RIP), that had been discussed before. Each step to recover radar signal perfectly through traditional CAMP algorithm needs at least five iteration of matrix multiplication, unlike the proposed adaptive recovery CAMP algorithm which doesn't need any iteration. On the other hand the proposed algorithm threshold calculation is more complex than the traditional threshold calculation.

\section{Conclusion}

This paper has introduced a general description about CS theory, the sparsity property of the radar signal, and focused on managing two main problems in the CS theory. Those problems are the limited number of reconstructed targets in received pulsed radar signal, and the complexity of the reconstruction process due to the iteration in the traditional approach. A suggested method has been proposed to solve these problems called adaptive recovery CAMP algorithm, based on calculating the threshold value in adaptively method according to the smallest of the noisy estimation of the pulsed radar signal, which has given better results.

The number of reconstructed targets using the proposed algorithm has been enhanced by 1.4 than the traditional one. The high complexity for threshold calculation in adaptively can be ignored with respect to the complexity of the traditional CAMP algorithm due to four reasons; the complexity of the five steps reconstruction in traditional algorithm opposite to three steps reconstruction in the proposed algorithm, the complexity of the number of iterations for reconstruction in traditional algorithm opposite to no iterations for reconstruction in the proposed algorithm, the traditional algorithm can recover the radar signal with approximately processing time 2.354 second, but the proposed algorithm can recover the radar signal with processing time 0.45 second. 
